\title{
Computing Wrench Cones for Planar Rigid Body Contact Tasks
}

\author{
Devin J. Balkcom * \\ Carnegie Mellon Robotics Institute \\ Pittsburgh, PA 15213. \\ devinari.cmu.edu
}

\author{
J.C. Trinkle ${ }^{\dagger}$ \\ Department of Computer Science \\ Rensselaer Polytechnic Institute \\ Troy, NY 12180-3590 \\ trink@cs.rpi.edu
}

\begin{abstract}
The contribution of this paper is the expansion of the range of possibilities in the analysis, planning, and control of contact tasks. The successful execution of any contact task fundamentally requires the application of wrenches (forces and moments) consistent with the task. We develop an algorithm for computing the entire set of external wrenches consistent with achieving a given augmented contact mode (e.g., sliding at contact 1 , rolling at contact 2 , and approaching potential contact 3 ) for one fixed and one moving part in the plane.

Unfortunately, because of the problem of frictional indeterminacy, it is usually not enough to determine the set of wrenches consistent with achieving a contact mode. Some of the computed wrenches may also be consistent with other undesirable contact modes. However, set operations on the cones returned by our algorithm can be designed to find the wrenches consistent only with desired contact modes.
\end{abstract}

This paper also presents some applications of the algorithm to analysis and planning problems. We show how to use set operations to compute the set of external wrenches with respect to which a fixtured part is 'strongly stable'. If the applied external wrench is in this set, the part is guaranteed not to move. We also show how the algorithm

* The work of this author was supported by a Department of Energy Computational Sciences Graduate Fellowship.

${ }^{\dagger}$ The work of this author was partially supported by the Laboratory Directed Research and Development program of Sandia National Laboratories. Sandia is a multi-program laboratory operated by Sandia Corporation, a Lockheed Martin Company, for the United States Department of Energy under Contract DE-AC04-94AL85000.

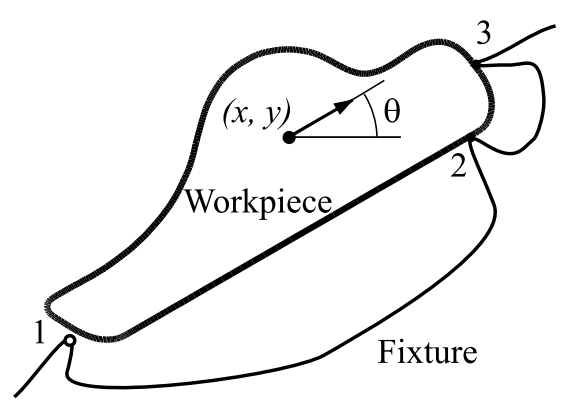

Figure 1: A workpiece nearly seated in a fixture.

may be used to create sensorless plans that guarantee that a workpiece will be correctly inserted into a fixture.

\section{Introduction}

One of the most basic operations in manufacturing is workpiece insertion. Consider the planar assembly task depicted in figure 1. The workpiece (a ratchet pawl) is to be fixtured for assembly. The goal is to achieve all three desired contacts (or fixels), so that the position and orientation of the workpiece will be uniquely determined. If the workpiece slides on fixels 2 and 3 while rotating counterclockwise, then the workpiece will eventually contact fixel 1.

This plan requires a device that applies specified wrenches. If the workpiece is positioned on a tray that is 
tilted to achieve the desired workpiece motion, then gravity provides the external wrench. If a spring is used to push the workpiece, then the deformation of the spring provides the wrench.

Regardless of the method used to apply the force, it is fundamental to the above plan that the set of external wrenches consistent with achieving the desired contact mode be known. In this paper, we present an algorithm to determine the set of external wrenches consistent with achieving any possible contact mode.

Unfortunately, due to the non-uniqueness problem inherent in most mathematical models of dynamic rigid body systems (see Lötstedt [13], for example), it is possible that the wrenches in a calculated set may be consistent not only with the desired mode, but also with another, undesirable mode. Therefore, we also show how unions and intersections of the sets of consistent external wrenches may be used to find wrenches consistent only with desired interactions between the workpiece and the fixture.

\subsection{Relation to previous work}

This paper collects and expands upon work by Balkcom and Trinkle presented in [3, 4]. Our approach is based on previous theoretical results in rigid body mechanics [17, 23] and complementarity theory [7]. In Pang and Trinkle [17], examples are presented in which the polyhedral convex cones of external wrenches consistent with achieving particular contact modes are calculated. We develop and extend their method into an algorithm that works with all contact modes. (We will refer to this algorithm as the wrench cone algorithm.) We represent polyhedral convex cones by matrices; our primary reference for operations on matrix representations of cones is Goldman and Tucker [11]. Hirai's Ph. D. thesis [12] describes an algorithm for conversion between representations of polyhedral convex cones that is somewhat more compuatationally efficient than that in [11], and we used Hirai's software as a component of the implementation of our work.

Brost [6] and Mason [14] provide good surveys of previous work on manipulation planning from the perspective of rigid body dynamics with Coulomb friction. Erdmann's work on generalized friction cones in configuration space $[8,9]$ provided one of the first methods for computing the possible motions of contacting rigid bodies under an applied wrench.

Prattichizzo and Bicchi [19] provides an analysis of the dynamic equations of rigid body contact problems from the perspective of linear controllability and observability. Like those authors, we consider a linear (and therefore local) model, analyzed at the current time. However, we focus on workpiece insertion (fixturing, or attaining a grasp), while Prattichizzo and Bicchi focus on the control of the workpiece after it has been grasped. Therefore, we consider contact interactions like sliding, separating, and approaching, which are indispensible for achieving contact, but which may be undesirable when manipulating a grasped object.

Apart from Pang and Trinkle [17], the work most similar to that presented here is probably Mason [14]. Mason describes a graphical method for finding the set of acceleration centers (and thus wrenches) consistent with achieving a particular contact mode. Our method is based on linear algebra rather than geometry; this allows more flexibility in the definition of contact modes. For example, the set of external wrenches consistent with maintaining contact at some point can be found, without specifying whether the mode involves sliding right, sliding left, or rolling. Another advantage of our algorithm is ease of implementation: the core of our sample implementation is about one hundred lines of simple $\mathrm{C}$ code.

We also use set operations on the calculated polyhedral convex cones to derive a set of wrenches consistent only with fixturing a planar workpiece. There have been many controller designs for rigid body insertion tasks; we will mention only a few characteristic approaches. As one example, McCarragher's petri-net controller [2, 15] sensed contact states by comparing sensor signals to a qualitative template derived from rigid body dynamic equations under the frictionless assumption. Then the manipulator applied controls consistent with certain desirable state transitions, and not with other state transitions.

Unlike McCarragher's controller, passive (or sensorless) controllers rely on a device designed to apply proper wrenches regardless of the current state. Erdmann and Mason develop a formal framework for the design of passive controllers in a paper on sensorless manipulation [10]. Whitney's remote center of compliance (RCC) [25] and Schimmels and Peshkin's accommodative wrist [22] are both examples of this approach. In the related problem of fixture clamp placement, Brost and Peters em- 
ployed a quasistatic analysis of the clamping process over the range of motion of the clamp's plunger with uncertain contact state [5].

Our fixturing method makes use of the presented wrench cone algorithm and is a passive controller. Generating the insertion strategy only requires taking unions and complements of some computed wrench cones, and is thus quite easy to implement. Like McCarragher, we assume that the state transitions are determined by a dynamic rather than a quasistatic rigid body model. Our approach to the well-known non-uniqueness problem of rigid body dynamics (for example, see [23, 24]) is to find a set of external wrenches consistent only with seating the workpiece. Although we assume that workpiece velocities are small, our method is also robust to sign changes in the tangential contact velocities.

\subsection{Structure of the paper}

Section 2 presents a mathematical model of our system of two planar rigid bodies, and makes necessary definitions. The model is composed of two parts: the NewtonEuler equations, and a set of constraints imposed by the choice of contact mode. It turns out that the set of external wrenches consistent with the equations and constraints is a polyhedral convex cone; section 3 reviews matrix representations of polyhedral convex cones. In section 4 , we consider a simple example of a desired contact mode, and show (by hand) how the implied constraints and the Newton-Euler equations may be combined to determine the consistent cone of external wrenches. Section 5 presents the complete wrench cone algorithm, and examples are given in section 6. Section 7 discusses the problem of frictional indeterminacy. We then show how the wrench cone algorithm may be used to find the cone of wrenches with respect to which a fixtured workpiece is strongly stable; that is, guaranteed not to move even in the presence of indeterminate contact forces. Sections 8 and 9 present an additional application of the wrench cone algorithm: a fixture insertion strategy that takes into account the problem of frictional indeterminacy.

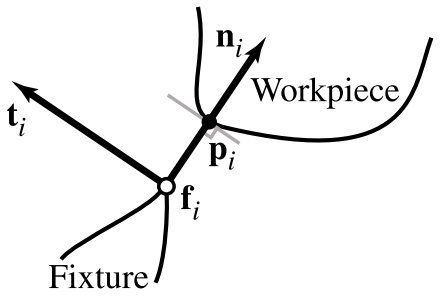

Figure 2: A local frame attached to fixel $i$.

\section{Mathematical model}

The instantaneous dynamic model of a system of rigid bodies with unilateral, frictional contacts can be formulated as a linear complementarity problem (LCP) [23] and solved by Lemke's algorithm [1]. The model consists of five components: the Newton-Euler equations, kinematic non-penetration constraints, a friction law, and normal and tangential complementarity conditions. Given the configurations and velocities of the moving bodies, the current set of contacts, the coefficients of friction, the inertia properties, and the applied external wrenches, the solution of the LCP yields contact forces and body accelerations satisfying all five components of the model.

Our problem may be seen as an inverse LCP. Given the rigid body configurations and a set of constraints on contact forces and accelerations imposed by the choice of contact mode, we want to find the set of consistent external wrenches. The derivation of our mathematical model therefore parallels that derived for LCP formulations. We begin by assuming that the workpiece is either touching or infinitesimally distant from each fixel, and that velocities are small enough that velocity product terms may be neglected from the dynamic equations.

\subsection{Variables and definitions}

Let $\mathbf{f}_{i}$ be the location of fixel $i$. Let $\mathbf{p}_{i}$ be the unique closest point on the workpiece to $\mathbf{f}_{i}$. We attach a right handed frame $\left(\mathbf{n}_{i}(t), \mathbf{t}_{i}(t)\right)$ to each fixel such that the first axis points at $\mathbf{p}_{i}$ (see figure 2 ). The signed distance, or gap, between the workpiece and fixel $i$ is $\left\langle\mathbf{n}_{i}(t),\left(\mathbf{p}_{i}-\mathbf{f}_{i}\right)\right\rangle$ where the angle brackets denote the usual inner product of vectors.

Let $v_{i \mathrm{n}}(t)$ and $v_{i \mathrm{t}}(t)$ denote the components of the ve- 
locity of $\mathbf{p}(i)$ in frame $i$ :

$$
\begin{aligned}
v_{i \mathrm{n}}(t) & =\left\langle\dot{\mathbf{p}}_{i}(t), \mathbf{n}_{i}(t)\right\rangle \\
v_{i \mathrm{t}}(t) & =\left\langle\dot{\mathbf{p}}_{i}(t), \mathbf{t}_{i}(t)\right\rangle .
\end{aligned}
$$

We now state four definitions. A contact state is the set of indices of fixels where contact has been achieved. A contact interaction is the relative motion at a point of contact: separating, rolling, sliding left, sliding right. A contact mode is the set of interactions at all the contacts. Because we are interested in insertion tasks, we define an additional contact interaction, 'approach'. We also extend the definition of a contact mode - an augmented contact mode is the extension of a contact mode that allows specification that the workpiece is approaching a nearby fixel. For example, the workpiece separates from fixel 1 and approaches fixel 2.

We may enumerate the possible contact interactions at fixel $i$ based on the distance of the fixel from the workpiece, and the normal and tangential components of the velocity of the closest point on the workpiece. For example, if the interaction at fixel $i$ is left sliding, then the gap is zero, $v_{i \mathrm{n}}=0$, and $v_{i \mathrm{t}}>0$. Table 1 enumerates the cases.

\begin{tabular}{|c|c|c|c|c|}
\hline Interaction & Abbrev. & gap & $v_{i \mathrm{n}}$ & $v_{i \mathrm{t}}$ \\
\hline \hline left sliding & $\mathrm{I}$ & 0 & 0 & $>0$ \\
right sliding & $\mathrm{r}$ & 0 & 0 & $<0$ \\
rolling & $\mathrm{n}$ & 0 & 0 & 0 \\
\hline approaching & $\mathrm{a}$ & $>0$ & $<0$ & - \\
\hline separating & $\mathrm{s}$ & $=0$ & $>0$ & - \\
& & $>0$ & $\geq 0$ & - \\
\hline
\end{tabular}

Table 1: Contact interactions.

Right sliding, left sliding, and rolling can occur only if contact has been achieved. Approaching can only occur if there is no contact, and would correspond to penetrating if contact had already been achieved. Separating may occur regardless of whether or not contact has been made.

We assume that the fixels have been ordered, and describe the augmented contact mode by a string, using the abbreviations from table 1. For example, the string 'als' should be read: the workpiece is approaching fixel 1 , sliding left over fixel 2, and separating from fixel 3 .

\subsection{Newton-Euler equations}

The Newton-Euler equations describe the dynamics of the system regardless of the contact mode. In this section we rearrange the these equations into a form that will be useful in later sections.

The net wrench $\mathbf{w}$ applied to the workpiece and the generalized acceleration $\dot{\nu}$ of the workpiece are related through the three-by-three inertia matrix $\mathbf{M}$ :

$$
\mathbf{w}=\mathbf{M} \dot{\nu} .
$$

Let $c_{i n}$ and $c_{i \mathrm{t}}$ be the normal and tangential components of the force applied to the workpiece by contact $i$. Assume there are $n$ contacts, and define $\mathbf{c}=\left[c_{1 \mathrm{n}} \ldots c_{n \mathrm{n}} c_{1 \mathrm{t}} \ldots c_{n \mathrm{t}}\right]^{T}$.

We partition $\mathbf{w}$ into the contribution of the contact forces $\mathcal{J} \mathbf{c}$ and that of external wrenches $\mathbf{g}$, and solve for the generalized acceleration of the workpiece:

$$
\dot{\nu}=\mathbf{M}^{-1}(\mathcal{J} \mathbf{c}+\mathbf{g}),
$$

where $\mathcal{J}$ is the Jacobian matrix (also known as the wrench matrix) that transforms the contact forces into the inertial frame and sums their moments about the center of mass of the workpiece.

Let $\mathbf{v}=\left[\begin{array}{llll}v_{1 \mathrm{n}} \ldots v_{n \mathrm{n}} & v_{1 \mathrm{t}} \ldots v_{n \mathrm{t}}\end{array}\right]^{T}=\mathcal{J}^{T} \nu$ be the vector of normal and tangential components of the contact velocities. Then the contact acceleration vector can be written as [18, 21]:

$$
\mathbf{a}=\frac{d}{d t} \mathbf{v}=\frac{d}{d t}\left(\mathcal{J}^{T} \nu\right)=\dot{\mathcal{J}}^{T} \nu+\mathcal{J}^{T} \dot{\nu}
$$

Premultiplying equation 4 by $\mathcal{J}^{T}$, assuming velocity product terms are negligible, and combining with equation 5 yields the Newton-Euler equations mapped into the contact frames:

$$
\mathbf{a}=\mathbf{A c}+\mathbf{B g}
$$

where $\mathbf{A}=\mathcal{J}^{T} \mathbf{M}^{-1} \mathcal{J}$ and $\mathbf{B}=\mathcal{J}^{T} \mathbf{M}^{-1}$.

We rearrange equation 6 to find a relation between external wrenches and the accelerations and forces at the contacts:

$$
\mathrm{Cy}=\mathrm{Bg}
$$

where

$$
\mathbf{C}=\left[\begin{array}{ll}
\mathbf{I}_{2 n \times 2 n} & -\mathbf{A}
\end{array}\right]
$$

is $(2 n \times 4 n)$ and

$$
\mathbf{y}=\left(\begin{array}{l}
\mathbf{a} \\
\mathbf{c}
\end{array}\right)
$$


has $4 n$ elements. Equation 7 will be the starting point for our algorithm to find the set of external wrenches consistent with a given contact mode.

\subsection{Constraints due to contact modes}

In this section we will discuss how the current contact state and the goal contact mode imply a set of constraints on $\mathbf{y}$, the vector of contact accelerations and forces.

Non-penetration Assume there is contact at fixel $i$. Then $a_{i n} \geq 0$; otherwise the fixel and the workpiece would interpenetrate.

Unilateral force Contact forces are unilateral: $c_{i n} \geq 0$.

Coulomb friction We denote the static and kinetic coefficients of friction by $\mu_{\mathrm{s}}$ and $\mu_{\mathrm{k}}$. If the workpiece is sliding to the right, then the frictional force will be on the left edge of the friction cone: $c_{i \mathrm{t}}=\mu_{\mathrm{k}} c_{i \mathrm{n}}$. If the workpiece is sliding to the left, then the frictional force will be on the right edge of the friction cone: $c_{i \mathrm{t}}=-\mu_{\mathrm{k}} c_{i \mathrm{n}}$. If the workpiece is not moving with respect to the fixture, then the friction force may fall anywhere in the static friction cone: $\left|c_{i \mathrm{t}}\right| \leq \mu_{\mathrm{s}} c_{i \mathrm{n}}$.

Separation If there is no contact between the workpiece and fixel $i$ or contact is breaking. Fixel $i$ cannot support a load, so $c_{i \mathrm{n}}=c_{i \mathrm{t}}=0$.

There are also constraints on the contact accelerations imposed by the choice of desired contact interaction at a fixel. If contact has been achieved, and we want the interaction to be left sliding, then we should choose $a_{i n}=$ 0 and $a_{i \mathrm{t}}<0$. We have collected the constraints on $\mathbf{y}$ implied by various contact interactions in table 2 .

\begin{tabular}{|c|c|c|c|c|}
\hline Abbrev. & $a_{i \mathrm{n}}$ & $a_{i \mathrm{t}}$ & $c_{i \mathrm{n}}$ & $c_{i \mathrm{t}}$ \\
\hline \hline $\mathrm{s}$ & $\geq 0$ & - & 0 & 0 \\
$\mathrm{a}$ & $<0$ & - & 0 & 0 \\
$\mathrm{u}$ & - & - & 0 & 0 \\
\hline $\mathrm{I}$ & 0 & $>0$ & $\geq 0$ & $-\mu_{\mathrm{k}} c_{i \mathrm{n}}$ \\
$\mathrm{r}$ & 0 & $<0$ & $\geq 0$ & $\mu_{\mathrm{k}} c_{i \mathrm{n}}$ \\
$\mathrm{n}$ & 0 & 0 & $\geq 0$ & $\left|c_{i \mathrm{t}}\right| \leq \mu_{\mathrm{s}} c_{i \mathrm{n}}$ \\
\hline $\mathrm{m}$ & 0 & - & $\geq 0$ & $\left|c_{i \mathrm{t}}\right| \leq \mu_{\mathrm{s}} c_{i \mathrm{n}}$ \\
\hline
\end{tabular}

Table 2: Constraints on elements of $\mathbf{y}$ due to contact interaction.
If the initial velocity of the workpiece is zero, then the constraints $\mathrm{S}, \mathrm{I}, \mathrm{r}$, and $\mathrm{n}$ correspond to the usual definition of contact modes. For example, assume we have a workpiece that is contacting three fixels, and initially at rest. If we apply an external wrench consistent with the constraints IIS, we expect the contact mode to be 'sliding left over fixel 1, sliding left over fixel 2, and separating from fixel 3' at the next time instant.

It is useful to define other constraints as well. Constraint a, 'approaching' is similar to s, but can only occur if there is no contact. Constraint $u$ implies that there is no contact, but does not constrain the part to accelerate towards or away from the fixel in question. The constraint $\mathrm{m}$, 'maintain', describes the situation where the normal acceleration is constrained so as to maintain contact, and the contact force is constrained to lie within the friction cone.

We will use $\mathcal{G}$ and a subscript to denote the set of external wrenches $\mathrm{g}$ satisfying a set of constraints. For example, the notation $\mathcal{G}_{\text {SS }}$ would describe the set of external wrenches consistent with the constraint that a given workpiece separates from two fixels. The algorithm we will describe in this paper can calculate a matrix describing the set of external wrenches consistent with the impending contact mode.

We will describe the current contact state by a binary number. State 00 describes the case where zero of two contacts have been achieved, and state 10 describes the case where contact had been achieved at the first fixel but not at the second.

\section{Matrix representations of polyhe- dral convex cones}

In this section, we review matrix representations of polyhedral convex cones. Our discussion is based on Goldman and Tucker [11]. Assume matrix F is given. The polar of $\mathbf{F}$ is defined as the set of solutions to the matrix inequality $\mathbf{F g} \leq 0$, where the inequality applies elementby-element:

$$
\operatorname{polar}(\mathbf{F})=\{\mathbf{g}: \mathbf{F g} \leq 0\}
$$

Any $\mathbf{g} \in \operatorname{polar}(\mathbf{F})$ makes a non-positive dot product with each row of $\mathbf{F}$. Each row of $\mathbf{F}$ may be interpreted as the 
normal to the plane bounding a half-space. This plane contains the origin and is included in the half-space described by the corresponding inequality. Thus solutions lie in the intersection of the half-spaces, and $\operatorname{polar}(\mathbf{F})$ is therefore a polyhedral convex cone with apex at the origin. We say that the inequality $\mathbf{F g} \leq 0$ is a face normal representation of the cone.

Similarly, assume matrix $\mathbf{G}$ is given and define the positive linear span of $\mathbf{G}$ :

$$
\operatorname{pos}(\mathbf{G})=\{\mathbf{g}: \mathbf{g}=\mathbf{G} \mathbf{z} \text { for some } \mathbf{z} \geq 0\} .
$$

Any vector $\mathbf{g} \in \operatorname{pos}(\mathbf{G})$ is in the positive linear span of the columns of $\mathbf{G}$, and we say that the equation $\mathbf{g}=\mathbf{G} \mathbf{z}$ together with the inequality constraint $\mathbf{z} \geq 0$ is a span representation of a polyhedral cone. The columns of $\mathbf{G}$ are referred to as generators.

\subsection{Converting between representations}

According to [11], Minkowski and Farkas first showed that for any face normal representation of a polyhedral convex cone, there is a corresponding span representation, and Weyl showed that the converse is true. If $\mathbf{F}$ or $\mathbf{G}$ is non-singular, then conversion between the two representations is easy and may be accomplished by matrix inversion. Goldman and Tucker [11] and Hirai [12] describe methods for performing the conversion if the matrix is rectangular or otherwise singular.

We introduce some new notation, and use the superscript $F$ to denote conversion from a span representation of a cone to a face normal representation. If we are given a matrix $\mathbf{H}$, then $\mathbf{H}^{F}$ refers to a matrix such that $\operatorname{pos}(\mathbf{H})=\operatorname{polar}\left(\mathbf{H}^{F}\right)$. This notation is particularly useful because it allows algebraic manipulation of a matrix equation and set of constraints, even in the case that the matrices involved are singular. The following theorem makes use of our notation.

Theorem 1 Assume we have a matrix equation and a set of constraints of the form:

$$
\begin{aligned}
\mathbf{K z} & =\mathbf{P g} \\
\mathbf{z} & \geq \mathbf{k}
\end{aligned}
$$

where $\mathbf{K}$ and $\mathbf{P}$ are constant matrices, $\mathbf{k}$ is a constant vector, and inequalities between vectors apply elementby-element. For a given vector $\mathbf{g}$, there exists $\mathbf{z}$ satisfying the equation and the inequality if and only if

$$
\mathbf{K}^{F} \mathbf{P g} \leq \mathbf{K}^{F} \mathbf{K} \mathbf{k}
$$

Proof: Define a change of variables $\mathbf{x}=\mathbf{z}-\mathbf{k}$. Then

$$
\begin{aligned}
\mathbf{z} & =\mathbf{x}+\mathbf{k} \\
\mathbf{K}(\mathbf{x}+\mathbf{k}) & =\mathbf{P g} \\
\mathbf{P g}-\mathbf{K} \mathbf{k} & =\mathbf{K} \mathbf{x} \\
\mathbf{x} & \geq 0 .
\end{aligned}
$$

The last two lines tell us that the vector $\mathbf{P g}-\mathbf{K k}$ lies in a polyhedral convex cone; this is the span representation of the cone. Therefore, we may convert to the face normal representation:

$$
\begin{aligned}
\mathbf{K}^{F}(\mathbf{P g}-\mathbf{K k}) & \leq 0 \\
\mathbf{K}^{F} \mathbf{P g} & \leq \mathbf{K}^{F} \mathbf{K} \mathbf{k} .
\end{aligned}
$$

Verification of the 'only if' condition is similar.

\section{A simple example}

Before presenting the complete algorithm, we present a simple example. Consider a disc-shaped workpiece (see figure 3) with unit radius, and inertia matrix $\mathbf{M}$ equal to the identity matrix. Fixel 1 is at the position $(0,-1)$, touching the workpiece (indicated by a small filled circle), and fixel 2 is slightly to the right of $(1,0)$, not quite touching the workpiece (indicated by a small unfilled circle). We want the workpiece to roll on fixel 1, and approach fixel 2; that is, the contact mode is 'na'. What external wrenches are consistent with this mode?

We first construct the Jacobian $\mathcal{J}$. Each column of $\mathcal{J}$ may be thought of as the wrench corresponding to a unit force applied to the workpiece at a point near a fixel, in a local coordinate direction.

$$
\begin{aligned}
\mathcal{J}=\left[\begin{array}{ccccc}
\mathbf{n}_{1} & \mathbf{n}_{2} & \mathbf{t}_{1} & \mathbf{t}_{2} \\
\mathbf{p}_{1} \times \mathbf{n}_{1} & \mathbf{p}_{2} \times \mathbf{n}_{2} & \mathbf{p}_{1} \times \mathbf{t}_{1} & \mathbf{p}_{2} \times \mathbf{t}_{2}
\end{array}\right] \\
=\left[\begin{array}{rrrr}
0 & -1 & -1 & 0 \\
1 & 0 & 0 & -1 \\
0 & 0 & -1 & -1
\end{array}\right] .
\end{aligned}
$$




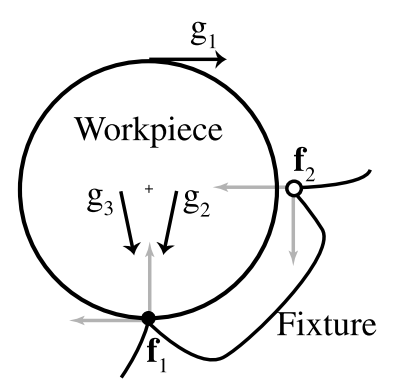

Figure 3: A simple example.

We calculate $\mathbf{C}$ and $\mathbf{B}$ and rewrite equation 7 :

$$
\left[\begin{array}{rrrrrrrr}
1 & 0 & 0 & 0 & -1 & 0 & 0 & 1 \\
0 & 1 & 0 & 0 & 0 & -1 & -1 & 0 \\
0 & 0 & 1 & 0 & 0 & -1 & -2 & -1 \\
0 & 0 & 0 & 1 & 1 & 0 & -1 & -2
\end{array}\right] \mathbf{y}=\left[\begin{array}{rrr}
0 & 1 & 0 \\
-1 & 0 & 0 \\
-1 & 0 & -1 \\
0 & -1 & -1
\end{array}\right] \mathbf{g}
$$

We now turn to the constraints on $\mathbf{y}$. Table 2 gives constraints on four elements of $\mathbf{y}$ for each interaction; we collect the constraints in table 3 .

\begin{tabular}{|c||c|c|c|c|c|}
\hline $\mathbf{y}$ & uncon & ineq & roll & slide & zero \\
\hline$a_{1 \mathrm{n}}$ & & & & & $=0$ \\
\hline$a_{2 \mathrm{n}}$ & & $<0$ & & & \\
\hline$a_{1 \mathrm{t}}$ & & & & & $=0$ \\
\hline$a_{2 \mathrm{t}}$ & $\mathrm{x}$ & & & & \\
\hline$c_{1 \mathrm{n}}$ & & $\geq 0$ & & & \\
\hline$c_{2 \mathrm{n}}$ & & & & & $=0$ \\
\hline$\left|c_{1 \mathrm{t}}\right|$ & & & $\leq \mu_{\mathrm{s}} c_{1 \mathrm{n}}$ & & \\
\hline$c_{2 \mathrm{t}}$ & & & & & $=0$ \\
\hline
\end{tabular}

Table 3: Constraints on $\mathbf{y}$ for contact mode 'na'.

We will take equation 12 , together with the constraints listed in table 3, and find a new equation and simpler constraints of the form:

$$
\mathbf{G z}=\mathbf{g}, \quad \mathbf{z} \geq 0
$$

1. Since $a_{1 \mathrm{n}}=a_{1 \mathrm{t}}=c_{2 \mathrm{n}}=c_{2 \mathrm{t}}=0$, we remove these elements from $\mathrm{y}$, and we remove the first, third, sixth, and eighth columns of $\mathbf{C}$.
2. Since $a_{2 n}$ is constrained to be less than zero, we change the signs of the elements of the second column of $\mathbf{C}$, replace $a_{2 \mathrm{n}}$ by $-a_{2 \mathrm{n}}$ in $\mathbf{y}$, and constrain $-a_{2 \mathrm{n}} \geq 0$. (For now, we ignore the issue posed by the strict inequality. This will be resolved in section 5.6.)

3. Presuming that $\mu_{\mathrm{s}}>0$, we replace the constraints $\left|c_{1 \mathrm{t}}\right| \leq \mu_{\mathrm{s}} c_{1 \mathrm{n}}$ by two equivalent constraints: $\left(\mu_{\mathrm{s}} c_{1 \mathrm{n}}+c_{1 \mathrm{t}}\right) / 2 \mu_{\mathrm{s}} \geq 0$ and $\left(\mu_{\mathrm{s}} c_{1 \mathrm{n}}-c_{1 \mathrm{t}}\right) / 2 \mu_{\mathrm{s}} \geq 0$. We make a variable substitution in $\mathbf{y}$, and take the appropriate linear combinations of columns five and seven of $\mathbf{C}$.

4. Since $a_{2 t}$ is unconstrained, we drop the equation involving it; we remove the fourth row of $\mathbf{C}$ and $\mathbf{B}$. Once this has been done, the fourth column of $\mathbf{C}$ is comprised only of zeros; we remove the column from $\mathbf{C}$ and $a_{2 \mathrm{t}}$ from $\mathbf{y}$.

After applying the above steps to equation 12, we have a new equation and set of constraints of the form used in theorem 1:

$$
\begin{aligned}
& {\left[\begin{array}{ccc}
0 & -1 & -1 \\
-1 & \mu_{\mathrm{s}} & -\mu_{\mathrm{s}} \\
0 & 2 \mu_{\mathrm{s}}-2 \mu_{\mathrm{s}}
\end{array}\right] }\left(\begin{array}{c}
-a_{2 \mathrm{n}} \\
\left(\mu_{\mathrm{s}} c_{1 \mathrm{n}}+c_{1 \mathrm{t}}\right) / 2 \mu_{\mathrm{s}} \\
\left(\mu_{\mathrm{s}} c_{1 \mathrm{n}}-c_{1 \mathrm{t}}\right) / 2 \mu_{\mathrm{s}}
\end{array}\right)=\left[\begin{array}{ccc}
0 & 1 & 0 \\
-1 & 0 & 0 \\
-1 & 0 & -1
\end{array}\right] \mathbf{g} \\
&\left(\begin{array}{c}
-a_{2 \mathrm{n}} \\
\left(\mu_{\mathrm{s}} c_{1 \mathrm{n}}+c_{1 \mathrm{t}}\right) / 2 \mu_{\mathrm{s}} \\
\left(\mu_{\mathrm{s}} c_{1 \mathrm{n}}-c_{1 \mathrm{t}}\right) / 2 \mu_{\mathrm{s}}
\end{array}\right) \geq 0
\end{aligned}
$$

where the matrices on the left and right sides of the equation are $\mathbf{K}$ and $\mathbf{P}$, respectively, and the vector on the left hand side is $\mathbf{z}$. If $\mu_{\mathrm{s}}=0.2$, and we solve for $\mathbf{g}$ by inverting $\mathbf{P}$ and premultiplying both sides of the equation, we arrive at the desired form:

$$
\begin{array}{r}
{\left[\begin{array}{rrr}
1.0 & -0.2 & 0.2 \\
0.0 & -1.0 & -1.0 \\
-1.0 & -0.2 & 0.2
\end{array}\right] \mathbf{z}=\mathbf{g}} \\
\mathbf{z} \geq 0 .
\end{array}
$$

This result is recognizable as a span representation of a polyhedral convex cone. A geometric interpretation is shown in figure 3 . The generators of $\mathbf{G}$ (its columns) will be denoted by $\mathbf{g}_{1}, \mathbf{g}_{2}$, and $\mathbf{g}_{3}$. Each generator corresponds to a wrench, which we may view as a directed line of force. The lines of force corresponding to $\mathrm{g}_{2}$ and $\mathrm{g}_{3}$ lie on the edges of the friction cone of fixel 1; the positive linear combinations of these generators are the external wrenches that may be balanced by the contact force 
at fixel 1. The line of force corresponding to $\mathbf{g}_{1}$ points to the right and is at the top of the disc; forces along this line will cause the disc to approach fixel 2, without breaking contact or generating a load at fixel 1 .

\section{The wrench cone algorithm}

We may generalize the procedure used in the example into an algorithm that works for any contact mode. First, we calculate the matrices $\mathbf{C}$ and $\mathbf{B}$. Once a contact mode has been chosen, table 2 may be used to determine the set of constraints on $\mathbf{y}$. We then build a series of matrices that may be used to transform the equation and constraints into a face representation of a polyhedral convex cone.

\subsection{Eliminate equations (Matrix E)}

Some elements of $\mathbf{y}$ may be unconstrained by the choice of augmented contact mode. We may eliminate the equations involving these variables by premultiplying $\mathbf{C}$ and $\mathbf{B}$ by a row selection matrix $\mathbf{E}$.

Let $\mathcal{E}$ be the set of indices of unconstrained elements of $\mathbf{y}$. We form $\mathbf{E}$ by removing the rows of $\mathbf{I}_{2 n \times 2 n}$ corresponding to elements of $\mathcal{E}$.

For the example problem discussed above, we examine the first column of table 3 ; the fourth variable $a_{2 \mathrm{t}}$ is unconstrained. Therefore, $\mathcal{E}=\{4\}$, and we form $\mathbf{E}$ by removing the fourth row of $\mathbf{I}_{4 \times 4}$.

\subsection{Negative variables (Matrix $\mathrm{N}$ )}

The choice of augmented contact mode may constrain some elements of $\mathbf{y}$ to be negative. We change the sign of columns of $\mathbf{C}$ so that all inequalities may be expressed using $>$ or $\geq$.

Let $\mathcal{N}$ be the indices of the elements of $\mathbf{y}$ constrained to be negative, and let $i_{i j}$ be the $(i, j)$ element of an appropriately sized identity matrix. We postmultiply $\mathbf{C}$ by the diagonal $4 n \times 4 n$ matrix $\mathbf{N}$ defined as follows:

$$
\begin{array}{ll}
n_{i j}=-1 & \text { if } i=j \text { and } i \in \mathcal{N} \\
n_{i j}=i_{i j} & \text { otherwise }
\end{array}
$$

For the example, we examine the second column of table 3 , and see that $\mathcal{N}=\{2\}$, since only $a_{2 \mathrm{n}}<0$. We form $\mathbf{N}$ by changing the sign on the second diagonal element of $\mathbf{I}_{8 \times 8}$.

\subsection{Rolling friction (Matrix R)}

If the augmented contact mode involves 'rolling' interactions, some elements of $y$ must satisfy constraints of the form $\left|c_{i \mathrm{t}}\right| \leq \mu_{\mathrm{s}} c_{i \mathrm{n}}$. We replace the variables $c_{i \mathrm{n}}$ and $c_{i \mathrm{t}}$ by $\left(\mu_{\mathrm{s}} c_{1 \mathrm{n}}+c_{1 \mathrm{t}}\right) / 2 \mu_{\mathrm{s}}$ and $\left(\mu_{\mathrm{s}} c_{1 \mathrm{n}}-c_{1 \mathrm{t}}\right) / 2 \mu_{\mathrm{s}}$, both of which are constrained to be non-negative. The variable substitution requires that we take appropriate linear combinations of columns of $\mathbf{C}$. We take the linear combination by postmultiplying $\mathbf{C}$ by a square matrix $\mathbf{R}$.

Let $\mathcal{R}$ be the indices of the elements $c_{i \mathrm{t}}$ of $\mathbf{y}$ that must satisfy rolling friction constraints. Define $\mathbf{R}$ to be the $4 n \times 4 n$ matrix with:

$$
\begin{array}{ll}
r_{i j}=\mu_{\mathrm{s}} & \text { if } i \in \mathcal{R} \text { and } i=j \\
r_{i j}=1 & \text { if } j \in \mathcal{R} \text { and } i=j-n \\
r_{i j}=-\mu_{\mathrm{s}} & \text { if } i \in \mathcal{R} \text { and } i=j+n \\
r_{i j}=i_{i j} & \text { otherwise }
\end{array}
$$

For our example, we examine the third column of table 3 , and find that the seventh element is subject to a rolling friction constraint. We build $\mathbf{R}$ from an $8 \times 8$ identity matrix, but set $r_{75}=-0.2, r_{57}=1$, and $r_{77}=0.2$.

\subsection{Sliding friction (Matrix S)}

If the augmented contact mode involves 'sliding' interactions, some elements of $\mathbf{y}$ must satisfy constraints of the form $c_{i \mathrm{t}}= \pm \mu_{\mathrm{k}} c_{i \mathrm{n}}$. We may eliminate $c_{i \mathrm{t}}$ by replacing a column of $\mathbf{C}$ by an appropriate linear combination of columns, and removing a column of $\mathbf{C}$. These operations may be accomplished by postmultiplying by a square matrix $\mathbf{S}$ and a column selection matrix $\mathbf{V}$ defined below.

Let $\mathcal{S}_{\mathrm{r}}$ be the indices of the elements of $\mathbf{y}$ constrained to be a positive multiple of another element. Let $\mathcal{S}_{1}$ be the indices of the elements of $\mathbf{y}$ constrained to be a negative multiple of another element. Define $\mathbf{S}$ to be the $4 n \times 4 n$ matrix with:

$$
\begin{array}{ll}
s_{i j}=\mu_{\mathrm{k}} & \text { if } i \in \mathcal{S}_{\mathrm{l}} \text { and } i=j+n \\
s_{i j}=-\mu_{\mathrm{k}} & \text { if } i \in \mathcal{S}_{\mathrm{r}} \text { and } i=j+n \\
s_{i j}=i_{i j} & \text { otherwise }
\end{array}
$$

For our example, we examine the fourth column of table 3 , and find that there are no sliding friction constraints, so $\mathbf{S}=\mathbf{I}_{8 \times 8}$. 


\subsection{Eliminate variables (Matrix V)}

We need to remove the columns of $\mathbf{C}$ corresponding to the variables $c_{i t}$ subject to sliding friction constraints. Similarly, some elements of $\mathbf{y}$ will be constrained to be 0 , and we may remove the corresponding columns of $\mathbf{C}$. Finally, since the unconstrained variables are accelerations appearing only in equations removed by the matrix $\mathbf{E}$, we may eliminate them. We may eliminate variables by postmultiplying by a column selection matrix $\mathbf{V}$.

Let $\mathcal{Z}$ be the indices of the elements of $\mathbf{y}$ constrained to be zero. Then define $\mathcal{V}=\mathcal{Z} \cup \mathcal{S}_{\mathrm{r}} \cup \mathcal{S}_{1} \cup \mathcal{E}$. We form $\mathbf{V}$ by removing any columns of $\mathbf{I}_{4 n \times 4 n}$ that have an index contained in $\mathcal{V}$.

For our example, we examine the first column of table 3, and find that the fourth element of $\mathbf{y}$ is unconstrained and may be eliminated. The fourth column of the table tells us that there are no sliding constraints, and the fifth column tells us that elements one, three, six, and eight are constrained to be zero and may be eliminated. We build $\mathbf{V}$ by removing the first, third, fourth, sixth, and eighth columns of $I_{8 \times 8}$.

\subsection{Cone form}

We apply all of the above matrices in order to equation 7 to find an equation and set of constraints of the form:

$$
\begin{aligned}
\mathbf{K z} & =\mathbf{P g} \\
\mathbf{z} & \geq 0
\end{aligned}
$$

where the inequality in 14 applies element-by-element and $\mathbf{K}, \mathbf{z}$, and $\mathbf{P}$ are defined as:

$$
\begin{aligned}
\mathbf{K} & =\mathbf{E C N R S V} \\
\mathbf{z} & =\mathbf{V}^{T} \mathbf{S}^{-1} \mathbf{R}^{-1} \mathbf{N}^{-1} \mathbf{y} \\
\mathbf{P} & =\mathbf{E B} .
\end{aligned}
$$

We may calculate $\mathbf{K}$ and $\mathbf{P}$; then equation 13 and inequality 14 'almost' give the cone containing the external wrench. In special cases, we may either take an inverse or pseudoinverse to find a cone form, as we did in the simple example. In general, we apply theorem 1 , choose $\mathbf{k}=0$ and define $\mathbf{F}=\mathbf{K}^{F} \mathbf{P}$. Then

$$
\mathbf{F g} \leq 0 .
$$

This is the face normal representation of the polyhedral convex cone containing the external wrench $g$.

So far, we have ignored the distinction between strict and non-strict inequalities in our discussions. Fortunately, theorem 1 allows strict inequalities to be converted to nonstrict inequalities by choosing $\mathbf{k}$ to have small positive elements, and considering limits as $\mathbf{k} \rightarrow 0$.

A related issue is that in the definitions of augmented contact modes in which the workpiece approaches fixels, we only required that accelerations towards fixels be nonnegative. For these modes, some solutions in the calculated cone may have zero or very small accelerations. This means that it could take a very long time for contact to be achieved. Minimum bounds on accelerations can be chosen if desired by setting appropriate elements of $\mathbf{k}$.

We summarize the algorithm as follows:

1. Calculate the Jacobian $\mathcal{J}$ and the matrices $\mathbf{C}$ and $\mathbf{B}$.

2. Determine the constraints on $\mathbf{y}$ implied by the mode.

3. Calculate the matrices $\mathbf{E}, \mathbf{N}, \mathbf{S}, \mathbf{R}$, and $\mathbf{V}$.

4. Calculate the matrices $\mathbf{K}$ and $\mathbf{P}$.

5. Find $\mathbf{K}^{F}$, as discussed in Section 3.

6. Calculate $\mathbf{F}=\mathbf{K}^{F} \mathbf{P}$.

A given wrench $\mathbf{g}$ is consistent with the contact mode if it satisfies $\mathbf{F g} \leq 0$.

\section{Implementation and examples}

We implemented the algorithm in $\mathrm{C}$, and used software described in Hirai [12] for the conversion between face normal and span representations of convex cones. Four example problems are shown in figure 4. For each example, the output of the algorithm was a matrix $\mathbf{F}$ of the form described above. In each case, we converted from face normal form to span form to find and display the wrench cone generators. Generators usually (but not always) describe degenerate or boundary solutions in the sense that a contact force or acceleration is zero that will be strictly positive when a force from inside the cone is applied.

Figure 4a shows an example for the contact mode 'lla'. The goal is to achieve contact at fixel 3 , while maintaining contact at fixels 1 and 2 . The generators $g_{1}$ and $g_{2}$ saturate the right edges of the friction cones, and $\mathrm{g}_{3}$ provides the negative torque about the center of rotation to cause 
a) mode 'lla'

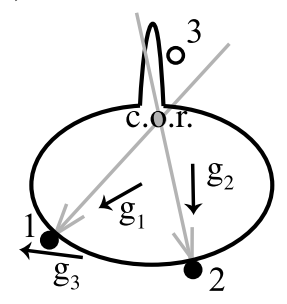

b) mode 'nnn'

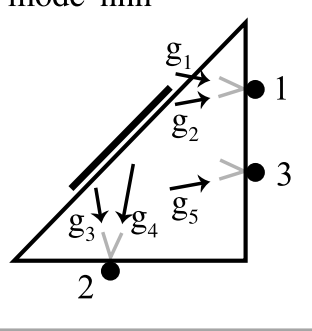

c) mode 'Ira'

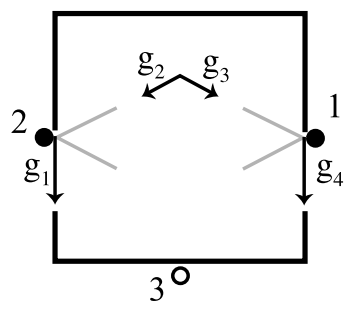

d) mode 'nn'

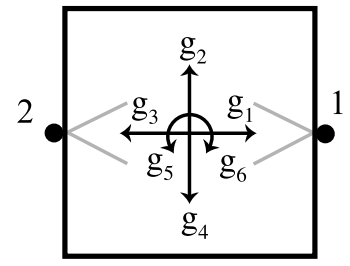

Figure 4: Four examples.

contact to be achieved at fixel 3. This example suggests how our algorithm might be used as part of a manipulation planner; one approach is described in section 8 .

Figure $4 \mathrm{~b}$ illustrates the problem of determining where a frictionless finger should be placed to achieve force closure. The problem can be solved using Reuleaux's graphical method [20]. Our algorithm finds the solution if we choose the contact mode 'nnn'. The thick black line on the surface of the workpiece shows places where the finger could be placed.

Figures $4 \mathrm{c}$ and $4 \mathrm{~d}$ show an example of the frictional indeterminacy problem that arises in a peg-in-hole insertion. There is a cone of wrenches consistent with seating the workpiece through mode 'Ira', but all of these wrenches are also consistent with the mode ' $n$ ', in which jamming occurs. (The wrench cone for figure $4 \mathrm{~d}$ has six generators. The two that are pure moments are drawn as arcs about the center of gravity.)

For a typical problem with three fixels, the average CPU time on a Pentium III system at $800 \mathrm{MHz}$ was about one millisecond, measured over one thousand executions of the algorithm. Table 4 shows the average CPU time for 1000 executions for problems with between one and six

\begin{tabular}{|c|l|}
\hline$n$ & CPU time \\
\hline 1 & $0.00003 \mathrm{~s}$ \\
2 & $0.00018 \mathrm{~s}$ \\
3 & $0.00101 \mathrm{~s}$ \\
4 & $0.00610 \mathrm{~s}$ \\
5 & $0.0363 \mathrm{~s}$ \\
6 & $0.2190 \mathrm{~s}$ \\
\hline
\end{tabular}

Table 4: Average CPU time, measured over 1000 executions.

fixels.

Profiling showed exponential algorithmic complexity underlying our cone computations (note the $6^{n}$ trend in the table). This was expected, because in the worst case, the number of cones to be considered when converting from span form to face normal form is known to be exponential in the number of contacts. (See Hirai [12] and Goldman and Tucker [11] for details of the conversion algorithm.) In the case of three fixels, $\approx 99.98 \%$ of the CPU time was spent on conversions between forms.

\section{Strong stability of a fixtured workpiece}

In the previous section, we discussed some examples where individual wrench cones consistent with achieving or maintaining a contact mode were derived. However, as we saw in figures $4 \mathrm{c}$ and $4 \mathrm{~d}$, wrench cones for different contact modes may overlap, causing an indeterminacy problem. In this section, we present an example of how set operations on wrench cones may be used to make guarantees about the interaction between the workpiece and the fixture.

Pang and Trinkle [17] make the following definitions:

Weak stability: There exists a solution to the rigid body dynamics model for which the acceleration of the workpiece is zero.

Strong stability: The acceleration of the workpiece is zero for all solutions of the rigid body dynamics model.

We say that a workpiece is weakly stable with respect 
to a set of external wrenches if for each wrench there is a solution with zero acceleration. Thus, weak stability is a generalization of force closure:

Force closure: The positive linear span of possible contact wrenches is all of wrench space.

The workpiece is in force closure if it is weakly stable with respect to all of wrench space.

The weak stability problem has been well studied. Take the set of unit wrenches corresponding to the edges of the friction cones; these wrenches are the edges of a polyhedral convex cone in wrench space. If the negative of the external wrench is included in this cone, then the part is weakly stable with respect to that wrench. Weak stability corresponds to the constraints nn (for two fixels) and nnn for three fixels. In figure $5, \mu_{\mathrm{s}}$ is large enough that each fixel is included in the friction cone of the other. Nguyen's condition for force closure is satisfied ([16]), and thus it is possible that any external wrench will be balanced by the contact forces. The weakly stable cone is all of wrench space.

If we want the part to be motionless in a rigid grasp or fixture, it is necessary that any applied external wrench be in the weakly stable cone. It is sufficient that the external wrench be in the strongly stable cone. We may calculate the strong stability cone by the following method:

1. Determine the kinematically feasible contact modes. (See Mason chapter 8 [14] for an algorithm based on Reuleaux's method [20].)

2. For each contact mode except $n$...n calculate the set of consistent wrenches. Take the complement of the union.

The workpiece is then strongly stable with respect to the calculated wrenches. Figure 5 shows an example. The workpiece is a disc touching two fixels. The kinematically feasible contact modes are ss, sl, sr, sn, Is, rs, ns, II, and rr; some corresponding rotation centers are shown on the figure. (Representative rotation centers corresponding to all kinematically consistent modes fall either on the lines tangent to the contacts, normal at the contacts, or in the cells created by the intersections of the half-spaces defined by these lines, as described in Mason [14].)

We calculate

$$
\mathcal{G}_{\text {strong }}=\overline{\mathcal{G}_{\mathrm{SS}} \cup \mathcal{G}_{\mathrm{Sl}} \cup \mathcal{G}_{\mathrm{Sr}} \cup \ldots \cup \mathcal{G}_{\mathrm{rr}}}
$$

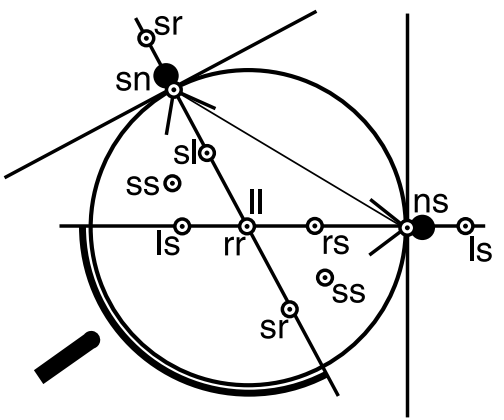

Figure 5: Computing strong stability.

Figure 5 shows the places where pushing with a frictionless finger would exert an external wrench in the strongly stable cone. The workpiece will not move if the finger pushes along the dark line. As shown in the figure, if $\mu_{\mathrm{s}}$ is large enough, each fixel will be included in the friction cone of the other: the weakly stable cone will be all of wrench space. The workpiece might not move if it is pushed somewhere other than along the dark line, but there are no guarantees!

Computation of the strongly stable cone also allows an analysis of situations where immobilization is to be achieved by a combination of external forces and geometric constraints (fixels). If $\mathcal{G}_{\text {strong }}$ has non-zero volume, and we apply a biasing wrench from the interior of $\mathcal{G}_{\text {strong, }}$, then the workpiece will not move under small disturbance wrenches of arbitrary direction.

We also point out that the first step of the algorithm, in which the kinematically feasible contact modes are determined, is only necessary from the standpoint of computational efficiency. There are four possible interactions at each contact: I, r, s, or $n$. So if we do not calculate the feasible contact modes, we must calculate $4^{n}-1$ wrench cones corresponding to each possible mode except $n$..n. For kinematically infeasible modes, the wrench cones computed will simply turn out to be empty, assuming the correct analysis has been done to preserve strict inequalities, as discussed in section 5.6.

The computation of strong stability involves finding the set of wrenches consistent only with the mode nn ...n. A similar approach can be used to find all wrenches consistent only with any other mode (for example II . . I, sliding 
left on all fixels), or to find wrenches consistent only with a given contact task. The next section will describe how unions and complements of wrench cones can be used to find the set of wrenches consistent only with workpiece insertion.

\section{Fixturing a workpiece}

The algorithm for calculating $\mathcal{G}_{\text {strong }}$ presented above is most useful as a tool for analysis of motionless rigid bodies. In this section, we consider the problem of planning an insertion task.

Consider figure 6a. The problem is to seat a discshaped workpiece of uniform density against the two fixels. The insertion process can be thought of as a finite state graph whose nodes represent the set of desired contacts that have been achieved. For example, if we call the desired contacts fixels 1, 2, and 3 (see figure 1), then one of the nodes of the transition graph will correspond to the case where contact has been achieved at fixels 2 and 3, but not at fixel 1. The goal state corresponds to the case where there is contact at all desired points. Transitions between the states occur when contacts are achieved or broken. We assume that the workpiece is quite close to both fixels initially and impacts can be ignored in the sense that they are dissipative, so that the workpiece may bounce off a fixel several times, but will eventually achieve steady contact with zero normal velocity.

By choosing constraints on contact forces and accelerations we may enable or disable state transitions. Consider the state transition graph shown in figure $7 \mathrm{~b}$. If there is no contact, then the initial state is 00 . If additionally the constraint au is satisfied, then the state transition $00 \rightarrow$ 10 is likely, since the constraint au ensures that the workpiece will accelerate towards the first fixel. Similarly, the constraints ua, aa, na, and an are all desirable, since they are consistent with state transitions that bring the system closer to the goal state.

It might seem that choosing an external wrench from the set

$$
\mathcal{G}_{\text {au }} \cap \mathcal{G}_{\text {ua }} \cap \mathcal{G}_{\text {aa }} \cap \mathcal{G}_{\text {na }} \cap \mathcal{G}_{\text {an }} \cap \mathcal{G}_{\text {nn }}
$$

would guarantee that the goal would be reached. However, the the wrench cone algorithm presented above only

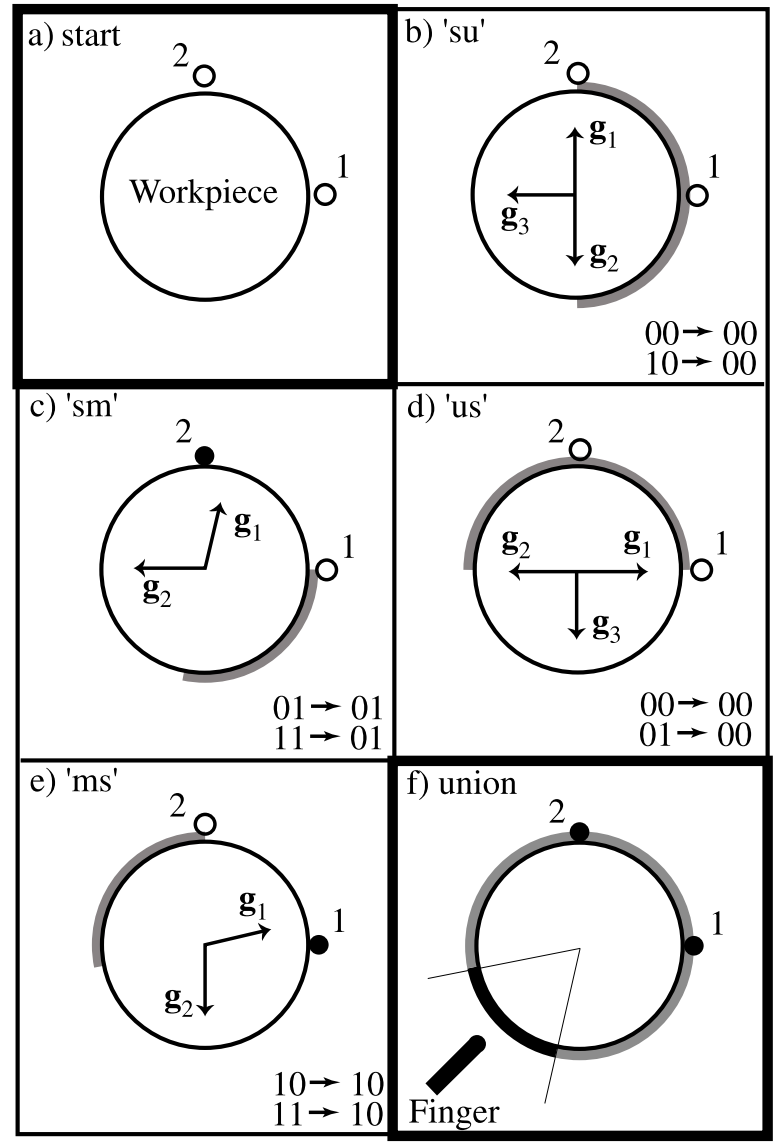

Figure 6: Finding the cone of wrenches that will cause a disc-shaped workpiece to contact two fixels, regardless of initial state.

finds the set of external wrenches consistent with constraints on the contact forces and accelerations. Due to the rigid body non-uniqueness problem (see [13] for example), it is possible that a single external wrench may be consistent with more than one vector of contact forces and accelerations, if contact has been achieved.

Therefore, instead of attempting to enable forwards transitions, we disable the backwards and self transitions by ensuring that the external wrench is not consistent with the constraints shown in figure 7c. Consider the state 00, where no contact has been achieved. If we apply a wrench not in the set $\mathcal{G}_{\mathrm{Su}}$, then the workpiece will accelerate to- 


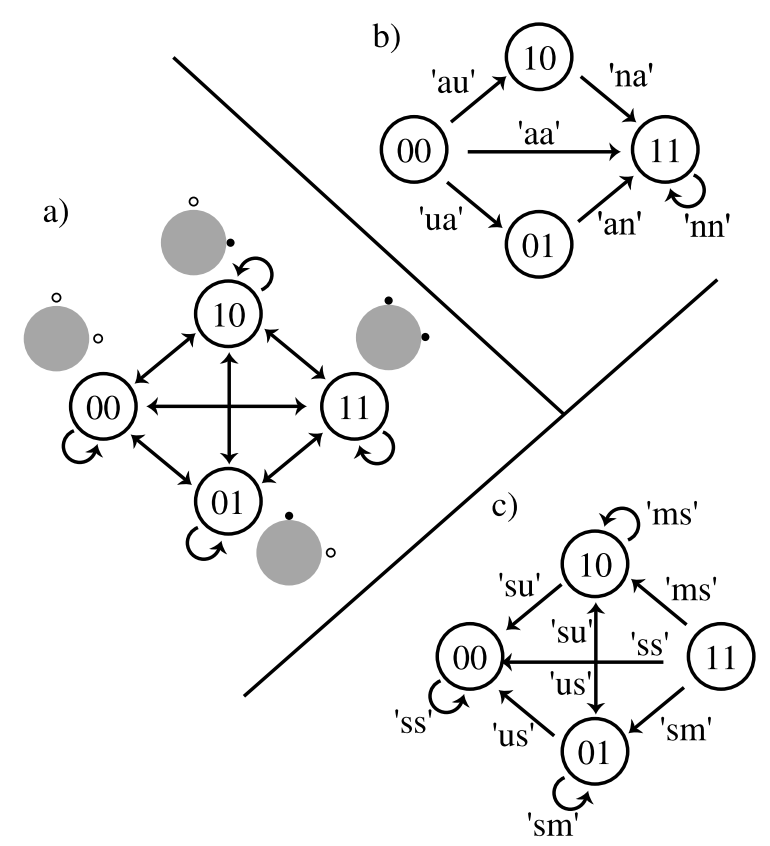

Figure 7: State transition graphs, two fixels.

wards the first fixel, and we expect the workpiece to eventually contact either fixel 1 or fixel 2. Analysis of the remaining state transitions is similar. If we choose an external wrench from the set

$$
\mathcal{G}_{\text {goal }}=\overline{\mathcal{G}_{\text {su }} \cup \mathcal{G} \text { us } \cup \mathcal{G}_{\mathrm{sm}} \cup \mathcal{G} \mathrm{ms}}
$$

then the number of contacts achieved will increase until the goal is achieved, regardless of the initial state.

Figures $6 \mathrm{~b}, 6 \mathrm{c}, 6 \mathrm{~d}$, and $6 \mathrm{e}$ show the external wrenches consistent with each of the backwards and self transitions. Since the workpiece is a disc, arbitrary torques may be applied about the center without causing the workpiece to approach or separate from the fixels. The consistent external forces are positive linear combinations of forces acting along the vectors shown. The thick gray lines show places on the surface of the part where pushing with a frictionless finger would generate these forces. Figure $6 f$ shows the union of these undesirable places to push (gray), and the complement of the union (black). Pushing on the black region will seat the workpiece, regardless of initial state.

It may seem surprising that we consider wrench sets

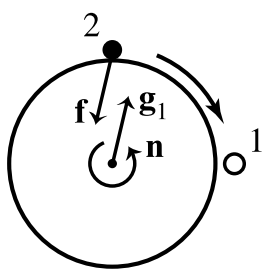

Figure 8: Spinning disc example.

involving the constraint $\mathrm{m}$, rather than the constraints $\mathrm{I}, \mathrm{r}$, and $\mathrm{n}$, corresponding to contact modes. It may also seem surprising that the pushing region shown in figure 6 is smaller than the strongly stable pushing region that could be calculated for this workpiece and fixture configuration. (In this case, the workpiece would be strongly stable with respect to any wrench generated by pushing with a frictionless finger anywhere in the third quadrant.)

The reason for these differences from what we might expect is that in the analysis of strong stability, we assumed that all velocities were zero, while for the fixture insertion strategy we only assume velocities are small enough to neglect velocity product terms from the Newton-Euler equations. Even if velocities are small, we must still consider the possibility that sign changes in the tangential velocities may change the direction of frictional forces.

The constraint $m$ implies that the normal component of acceleration be zero for the fixel in question, but does not constrain the direction of the tangential acceleration or of the tangential contact force. Therefore, for a single fixel with positive friction coefficient,

$$
\mathcal{G}_{\mathrm{m}} \supset \mathcal{G}_{\mid} \cup \mathcal{G}_{\mathrm{r}} \cup \mathcal{G}_{\mathrm{n}}
$$

Calculating $\mathcal{G}_{\mathrm{Sm}}$ and $\mathcal{G}_{\mathrm{ms}}$ rather than $\mathcal{G}_{\mathrm{Sl}}, \mathcal{G}_{\mathrm{Sr}}, \mathcal{G}_{\mathrm{Sn}}$, $\mathcal{G}_{\text {IS }}, \mathcal{G}_{\text {rs }}$, and $\mathcal{G}_{\text {ns }}$ saves some computation. There is an additional advantage: using the constraint $\mathrm{m}$ makes the algorithm robust to sign changes in the tangential velocities. As a concrete example, consider figure 6c. It might seem that a force applied along $\mathbf{g}_{1}$ would ensure that contact would be made at fixels 1 and 2 , since

$$
\begin{aligned}
& \mathbf{g}_{1} \notin \mathcal{G}_{\text {Sl }} \cup \mathcal{G}_{\text {Sr }} \cup \mathcal{G}_{\text {Sn }} \\
& \mathbf{g}_{1} \in \mathcal{G}_{\text {strong }} .
\end{aligned}
$$


However, if there are non-zero velocities, then there is a case where applying a force $\mathbf{g}_{1}$ will not cause acceleration of the workpiece towards fixel 1. Figure 8 shows an example, constructed from figure $6 \mathrm{c}$. The workpiece is initially spinning clockwise slowly. Therefore the force applied by fixel 2 is along an edge of the friction cone, labelled $\mathbf{f}$ in the figure. If we apply a force along $\mathbf{g}_{1}$, the total wrench may be just a positive torque $\mathbf{n}$ around the center of the disc; the workpiece will not accelerate towards fixel 1. Since calculating the sets $\mathcal{G}_{\mathrm{Sm}}$ and $\mathcal{G}_{\mathrm{ms}}$ does not require an assumption about what side of the friction cone the contact force will lie on, this case is correctly handled and the sensorless plan described above is robust to sign changes in the tangential contact velocities.

What will happen once the goal has been reached? Examining the component polyhedral convex cones of $\mathcal{G}_{\text {goal }}$ and $\mathcal{G}_{\text {strong }}$ shows that for any number of fixels

$$
\mathcal{G}_{\text {goal }} \subset \mathcal{G}_{\text {strong }}
$$

Therefore, a wrench from the interior of $\mathcal{G}_{\text {goal }}$ can act as a biasing wrench that will guarantee that the workpiece will not move after fixturing. If the workpiece is seated by a constant wrench (applied by gravity or a spring-loaded finger, for example), then disturbance wrenches of less than a certain magnitude will not move the workpiece after it has been seated. The magnitude of the permitted disturbance wrench may be calculated from the biasing wrench applied and the shape of the strongly stable cone. A conservative estimate of the magnitude might be calculated by considering the radius of the largest ball that would fit in the cone, centered on the biasing wrench.

\section{Achieving three contacts}

A similar strategy may be applied if three contacts are to be achieved. Figure 9 shows a state transition graph that guarantees that the workpiece will be seated. In order to ensure that the wrench applied is consistent only with this state transition graph and with no others, we consider all possible state transitions. We summarize the results in tabular form:

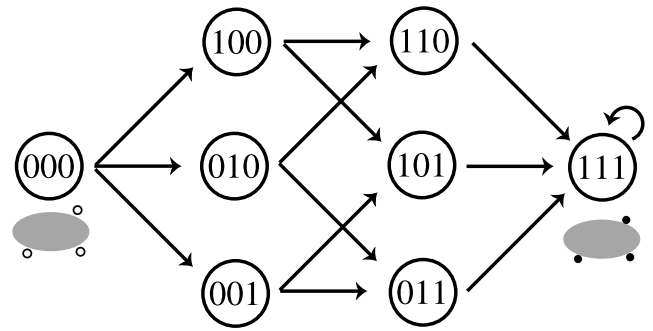

Figure 9: State transition graph, three fixels.

\begin{tabular}{|c|c|}
\hline State & Constraints \\
\hline 000 & sss \\
\hline 100 & suu, mss \\
010 & usu, sms \\
001 & uus, ssm \\
\hline 110 & ssu, msu, smu, $\mathbf{~ m m s}$ \\
101 & sus, mus, sum, msm \\
011 & uss, ums, usm, smm \\
\hline 111 & sss, mss, sms, mss, mms, smm, msm \\
\hline
\end{tabular}

Some of the constraints listed are redundant. For example, if a wrench is in the set $\overline{\mathcal{G}_{\text {SuU }}}$, then it is also in the set $\overline{\mathcal{G}_{\text {SSS }}}$. In all, twelve sets must be computed, corresponding to the constraints shown in bold. Wrenches in the complement of the union of these sets ensure that the goal will be reached. Figure 10 shows two examples generated by our sample implementation of the algorithm. The friction coefficient $\mu$ was chosen to be 0.2 in each case. As long as the velocity product terms in the dynamic equations are negligible, pushing along the thick black curve with a frictionless finger will seat the workpiece, regardless of initial state.

Figure 11 shows the dependency of $\mathcal{G}_{\text {goal }}$ on the friction coefficient. For this example, $\mathcal{G}_{\text {goal }}$ is fairly large when $\mu=0.01$, but quite small when $\mu=0.6$. This is what we might expect, since the workpiece seems more likely to become jammed in the fixture in the presence of high friction. Unfortunately, since the conversion between span and face representations of polyhedral convex cones used in the last step of the wrench cone algorithm is numerical, it is difficult to determine the dependence analytically unless either $\mathbf{K}$ or $\mathbf{P}$ is non-singular. 

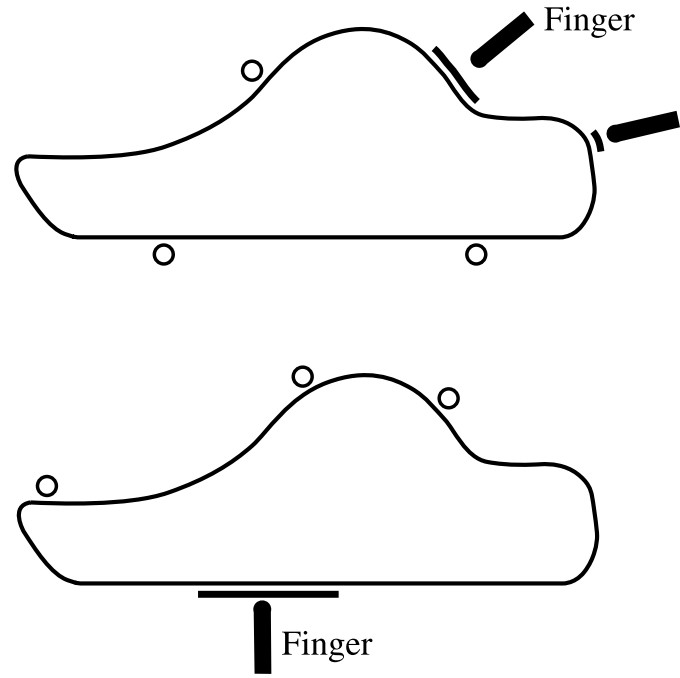

Figure 10: A frictionless finger seating a workpiece against three fixels.

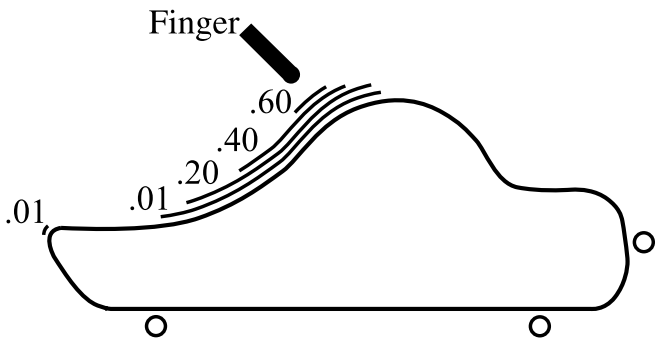

Figure 11: Sensorless plans for friction coefficients 0.01 , $0.20,0.40$, and 0.60 .

Finally, we point out that the fixturing approach described is quite conservative. The state transition graphs shown in figures 7 and 9 are not the only state transition graphs that guarantee that all desired contacts will be achieved. A complete approach would enumerate all acyclic state transition graphs with one sink (the goal state) and $2^{n}-1$ sources (the non-goal states). The corresponding wrenches would be calculated for each graph and the result would be the union of the wrenches.

\section{Limitations and future work}

In this section, we describe some of the limitations of the algorithms presented, and discuss how they might be addressed by future work. We discuss three primary issues: the worst-case computational complexity, assumptions that may cause the algorithms to be overly conservative, and other limiting assumptions.

The wrenchcone algorithm has exponential complexity, since the underlying conversion between representations of polyhedral convex cones is exponential. This fundamentally limits the number of fixels. From the perspective of fixture design and other similar problems, this may not be a significant limitation. If there are more than three fixels in the planar case, or more than six fixels in a 3D case (not discussed in this paper), then the system is overconstrained, and it is not possible to guarantee that all contacts are simultaneously achieved without a model of compliance.

Nonetheless, more efficient algorithms might be useful for an analysis of general contact problems. One possible approach might be to calculate whether specific individual wrenches were contained in a desired polyhedral convex cone. Such an algorithm could probably be made particularly efficient in the case where $\mathbf{K}$ or $\mathbf{P}$ were sparse and non-singular. Although the wrenchcone algorithm is itself exponential, we point out that the number of wrench cones that must be calculated to find the strongly stable cone is only polynomial in the number of contacts. (See Mason [14], for example.)

Another limitation is the possibly over-conservative nature of the sensorless insertion strategy. The intended use of our algorithm was fixture design; the goal was to design fixtures in such a way that a very simple control strategy could be applied to insert parts in a robust fashion. There are many workpiece shapes and fixel locations for which $\mathcal{G}_{\text {goal }}$ is in fact empty. More complicated control strategies involving sensing or time-varying wrenches could probably also be designed or analyzed using the wrenchcone algorithm.

Finally, we point out that our algorithm is only suitable for local, instantaneous analysis of planar contact tasks. We neglected velocity product terms for simplicity. It turns out that velocity product terms only shift the cones computed, and could be easily handled by the wrenchcone algorithm. The extension to three dimensions is more dif- 
ficult, since the friction cones become non-linear. The typical approach (used in LCP formulations, for example) is to linearize the friction cones. Although a slight extension of the wrenchcone algorithm could calculate the wrenches consistent with a linearized contact mode, the large number of modes would make computing $\mathcal{G}_{\text {strong }}$ computationally infeasible. Finding computationally efficient ways to calculate a conservative subset of $\mathcal{G}_{\text {strong }}$ is a possible direction of future work.

\section{Conclusion}

We developed an algorithm to find the polyhedral convex cone of external wrenches consistent with achieving a contact mode between two rigid bodies, one fixed and one moveable. The formulation of the model closely follows the formulation of the rigid body dynamics problem as a linear complementarity problem, and we also used results from the theory of polyhedral convex cones. We implemented the algorithm and presented some example results.

Additionally, we showed how to compute the cone of external wrenches with respect to which two contacting rigid bodies are strongly stable. Finally, we presented a method to determine a set of external wrenches consistent only with state transitions that increase the number of contacts. This method explicitly avoids the problem of non-uniqueness of solutions to the rigid body dynamics problems with Coulomb friction. The wrench set derived to seat the workpiece is a subset of the strongly stable cone.

\section{Acknowledgment}

The authors wish to thank Jong-Shi Pang, Matthew T. Mason, and Eric J. Gottlieb for their technical guidance and suggestions.

\section{References}

[1] M. Anitescu and F. Potra. Formulating multi-rigidbody contact problems with friction as solvable linear complementarity problems. ASME Journal of Nonlinear Dynamics, 14:231-247, 1997.
[2] D. Austin and B. McCarragher. Robust discrete event controller synthesis for constrained motion systems. In Symposium on Robot Control, pages 801-807. Elsevier, September 1997.

[3] D. Balkcom, E. Gottlieb, and J. Trinkle. A sensorless insertion strategy for rigid planar parts. In Proceedings, IEEE International Conference on Robotics and Automation, pages 882-887, 2002.

[4] D. Balkcom, J. Trinkle, and E. Gottlieb. Computing wrench cones for planar contact tasks. In Proceedings, IEEE International Conference on Robotics and Automation, pages 869-875, 2002.

[5] R. Brost and R. Peters. Automatic design of 3d fixtures and assembly pallets. Technical Report SAND95-2411, Sandia National Laboratories, January 1997.

[6] R. C. Brost. Analysis and Planning of Planar Manipulation Tasks. PhD thesis, Carnegie Mellon University School of Computer Science, January 1991.

[7] R. W. Cottle, J. Pang, and R. E. Stone. The Linear Complementarity Problem. Academic Press, 1992.

[8] M. A. Erdmann. On motion planning with uncertainty. Master's thesis, MIT Department of Electrical Engineering and Computer Science, August 1984.

[9] M. A. Erdmann. On a representation of friction in configuration space. International Journal of Robotics Research, 13(3):240-271, June 1994.

[10] M. A. Erdmann and M. T. Mason. An exploration of sensorless manipulation. IEEE Journal of Robotics and Automation, 4(4):369-379, August 1988.

[11] A. J. Goldman and A. W. Tucker. Polyhedral convex cones. In H. W. Kuhn and A. W. Tucker, editors, Linear Inequalities and Related Systems, pages 1940. Princeton Univ., York, 1956.

[12] S. Hirai. Analysis and Planning of Manipulation Using the Theory of Polyhedral Convex Cones. $\mathrm{PhD}$ thesis, Kyoto University, March 1991. 
[13] P. Lötstedt. Mechanical systems of rigid bodies subject to unilateral constraints. SIAM Journal of Applied Mathematics, 42(2):281-296, 1982.

[14] M. T. Mason. Mechanics of robotic manipulation. MIT Press, 2001.

[15] B. McCarragher and H. Asada. A discrete event controller using petri nets applied to assembly. In Proceedings, IEEE International Conference on Intelligent Robots and Systems, pages 2087-2094, 1992.

[16] V. Nguyen. Constructing force-closure grasps. International Journal of Robotics Research, 7(3):316, June 1988.

[17] J. Pang and J. Trinkle. Stability characterizations of rigid body contact problems with coulomb friction. Zeitschrift für Angewandte Mathematik und Mechanik, 80(10):643-663, 2000.

[18] F. Pfeiffer and C. Glocker. Multibody Dynamics with Unilateral Contacts. Wiley, 1996.

[19] D. Prattichizzo and A. Bicchi. Dynamics analysis of mobility and graspability of general manipulation systems. IEEE Transactions on Robotics and Automation, 14(2), April 1998.

[20] F. Reuleaux. The Kinematics of Machinery. Macmillan, 1876. Republished by Dover, New York, 1963.

[21] N. Sarkar, X. Yun, and V. Kumar. Dynamic control of 3-D rolling contacts in two-arm manipulation. IEEE Transactions on Robotics and Automation, 13(3):364-376, 1997.

[22] J. M. Schimmels and M. A. Peshkin. Force assembly with friction. IEEE Transactions on Robotics and Automation, 10(4):465-497, 1994.

[23] J. Trinkle, J. Pang, S. Sudarsky, and G. Lo. On dynamic multi-rigid-body contact problems with coulomb friction. Zeitschrift für Angewandte Mathematik und Mechanik, 77(4):267-279, 1997.

[24] J. Trinkle and D. Zeng. Prediction of the quasistatic planar motion of a contacted rigid body. IEEE Transactions on Robotics and Automation, 11(2):229-246, April 1995.
[25] D. E. Whitney. Quasi-static assembly of compliantly supported rigid parts. Journal of Dynamic Systems, Measurement, and Control, 104:65-77, March 1982. 